\title{
CURRENT TRENDS IN THE TREATMENT OF VENEREAL DISEASE IN THE UNITED STATES OF AMERICA*
}

\author{
By R. R. WILLCOX, M.B., B.S. \\ Lieutenant-Colonel, R.A.M.C. \\ Adviser in Venereology to the War Office
}

On 7 th and 8th February 1946 the United States Public Health Service held a meeting of the penicillin investigators of the National Research Council, at Washington (D.C.). On the 9th of February there was also held, at Bethesda in Maryland, the first Regional Seminar on Penicillin Therapy in the Venereal Disease Programme of the United States Public Health Service. It was my privilege to attend both these meetings as an observer, and subsequently to visit clinics and meet some of the leading authorities on venereal disease in the cities of Washington (D.C.), Baltimore, Philadelphia (Pa.), New York and Chicago. The information gained as a result of these contacts is the basis of this paper.

\section{General impressions}

In some places the treatment of gonorrhoea is still divorced from that of syphilis, the latter disease being treated in dermatological clinics and the former in urology centres (as in the Hospital of the University of Pennsylvania). In other places, such as the Johns Hopkins Hospital, Baltimore, both diseases are treated together. In municipal health centres and venereal diseases clinics, patients with both disorders are seen in the first place (as in New York and Chicago), but whereas gonorrhoea is dealt with in the clinics, patients with syphilis are transferred to the United States Public Health Rapid Treatment Centre for in-patient penicillin treatment. Patients with soft sore (chancroid), lymphogranuloma inguinale and granuloma venereum tend to be grouped with those having syphilis, whereas patients with semi-venereal conditions (non-specific urethritis, penile warts and so forth) are frequently referred to the urological or surgical departments. Venereal disease - or at any rate syphilis -is usually notifiable to the city health departments, which undertake also the "chasing'" of contacts. Repeated letters, registered letters, telegrams and special visitors are all used to bring the patients in. Propaganda is much in evidence ; most of the trams in Chicago, for example, carry venereal disease placards, and press and radio are also employed. Matches, too, which are given away free with cigarettes in the United States, are used in this way. Defaulters are usually contacted in the first instance by the clinic concerned. One (Belle Vue Hospital, New York) relies on a battery of letters in recalcitrant cases; these are sent simultaneously to the patient, his home, his work, his trade union and his known relatives. Many clinics never discharge a syphilitic patient. Patients who refuse to attend may be compelled to do so, ultimately, by the City Health Department. Venereal disease control officers have large staffs dealing with notification and defaulter contact tracing. Chicago, which undertakes more propaganda than do most places, keeps an eye open for sources of infection, and if a particular bar or hotel is suspected of being a hotbed of disease, the proprietor is visited and asked to mend his ways. If he fails to cooperate, his business may be closed by the Health Department, and notices such as "Venereal Disease: Beware", and "Syphilis: Keep Out" may be plastered on his premises. This has been done only a few times; hotel keepers usually cooperate.

\section{Gonorrhoea}

It was an often-expressed view that "gonorrhoea was beaten", and, although there were isolated voices urging maintained caution, there was an increasing tendency to relax the severity of the tests of cure of the disease.

*An address to the Medical Society for the study of Venereal Diseases, 30th March, 1946. 


\section{TREATMENT OF VENEREAL DISEASE IN THE UNITED STATES}

Treatment.- Treatment was with aqueous penicillin, or with penicillin given in oil and beeswax or by other delaying methods, usually on an ambulatory basis. The dosage employed in the 6 clinics visited which treated gonorrhoea was, with one exception, 200,000 units ; in the exceptional case it was 300,000 units. Three of the clinics used the aqueous solution, the dosage consisting of 4-5 doses at 2-3 hours' intervals to a total of 200,000 units. Two others used penicillin in beeswax in single doses of 200,000 and 300,000 units, and one gave 200,000 units of calcium penicillin in a cholesterol preparation in peanut oil (arachis oil). Excellent results were claimed with all the methods employed.

Tests of cure (males). - Tests of cure were generally made early. The Army had its men seen once a week, for inspection, for 3 weeks only, and a final Kahn test only is done at the end of 3 months. At the Johns Hopkins Clinic male patients attended once a week for 3 weeks for prostatic massage and urine culture, after which time they were seen once a month for 4 months for blood tests, in order to exclude syphilis. Other clinics reduced their tests still further, doing a culture at the end of 24 hours and repeating it at the end of 5 and 10 days, whereas in a very large out-patient clinic in Chicago, which was using penicillin in oil and beeswax, a prostatic massage and culture were done after 3 days and were followed by a blood test only, once a month for 3 months. At the Walter Reed Hospital, Washington, where Romansky's oil-beeswax penicillin was in use, 300,000 units were given, and the patients were watched without laboratory tests for one week. At the United States Marine Hospital, Staten Island (N.Y.), Dr. J. F. Mahoney gave 200,000 units of aqueous solution in 5 doses and, apart from an instruction to the men-to obtain a blood test at the end of 3 months, there was no provision at all for tests of cure. This procedure was based on a large-scale investigation, consisting of culture on alternate days for 3 weeks, in which the number of failures had proved to be negligible in relation to the large numbers treated. Some 400 men are being treated each month by this method. In the Canadian Army in Canada, 200,000 units of penicillin in an aqueous solution, given in 4 doses, was the treatment ; inspections were made 3 times in the first 2 weeks and followed by a blood test at the end of 3 months.

Tests of cure (females).- Greater caution is being observed with females, with general examination of the order of once a month for 3 months, although, at the very large clinic in Chicago, cultures and smears after 3 days constituted the only follow-up, apart from monthly blood tests for 3 months in satisfactory cases. The principle of treating clinically doubtful or even negative female contacts of infected men, on epidemiological grounds, was adopted largely.

Research.- Dr. Mahoney described his work with 300 prisoner volunteers, in whom he instilled, per urethrum, strains of gonococci. Only 30 per cent became infected. At most of the centres which I visited some small-scale experiments of between 20 and 30 cases had been treated experimentally with penicillin orally. All the investigators seem to have been satisfied that such therapy was possible and not to have pursued the matter farther.

\section{Syphilis}

The current views on the treatment of syphilis were expressed at the United States Public Health Service and National Research Council meeting held at Washington on 7 th and 8th February, at which Dr. J. E. Moore presided.

If the conclusions reached appear to be uncrystallized, it is because, for some years now, most of the leading clinics have been participating in a nation-wide research into the action of pencillin on syphilis and this work is still very far from complete. This research, which was taken over in 1946 by the United States Public Health Service, costs about $\$ 1,000,000$ a year. It is directed by an advisory committee, the members of which are Dr. J. E. Moore (Chairman), Dr. J. F. Mahoney, an Army, a Navy and a Public Health Service representative, two clinicians (Dr. J. H. Stokes and Dr. H. Solomon) and a biostatistician (Dr. Reed). I was privileged to attend some meetings of this committee as an observer. 


\section{THE BRITISH JOURNAL OF VENEREAL DISEASES}

Some 43 of the leading clinics and 6 laboratories participate in the scheme, treating syphilis according to prescribed schedules and sending their data to the Department of Biostatistics at the Johns Hopkins University for analysis. Data were presented up to August 1945 on more than 10,000 cases of early syphilis treated in accordance with one of the official schedules.

\section{Prenatal syphilis}

Dr. N. R. Ingraham (Philadelphia) described the results of treating 79 pregnant women suffering from early syphilis and 35 from latent syphilis with doses of penicillin between $1,200,000$ and $2,400,000$ units, given over $7 \frac{1}{2}$ days. The success rate of congenital syphilis prevention of over 97 per cent was claimed to be unequalled by any other form of therapy, there being only 3 abortions and one case of congenital syphilis in both series In a quarter of the number of cases treatment began as late, as the 32nd week, and it was concluded that penicillin was effective at any stage of the pregnancy. Only 16 per cent of the mothers had become seronegative at the time of delivery, whereas 27 per cent of the apparently normal infants were seropositive at birth. These latter usually became seronegative within a month without treatment, although one patient took as long as 72 days.

\section{Congenital syphilis}

Dr. Platou (Tulane University) described 191 cases treated at a mean age of 164 days. Sodium penicillin was given as either 20,000-40,000 units per kilogram in 60 three-hourly injections, or 80,000 units per kilogram in 120 equal injections spread over 15 days. Reactions were gratifyingly insignificant. Healing of visible lesions was rapid, but that of extensive osseous involvement took 3-5 months. Clinical and serological relapse together were encountered 5 times and serological relapse alone 7 times; 24 deaths occurred during treatment but were attributed to childish disorders. It was concluded that penicillin was the best agent so far employed for the treatment of congenital syphilis.

\section{Early syphilis}

The cumulative observations of the result of the participating clinics have been analysed by the Central Statistical Unit of the Johns Hopkins University and were presented by Dr. Rosahn in the form of a series of graphs.

\section{Peńicillin and other treatiment compared.-Dr. Rosahn's conclusions were as follows.}

(1) Total dosage. - The cumulative proportion of failure at 11 months varied from 15 per cent $(2,400,000$ units) to 62 per cent with 60,000 units of penicillin.

(2) Combined treatment: (a) Penicillin and arsenic.- The cumulative percentage of failure at the end of 11 months with a total dose of 300,000 units of penicillin was twice as great when it was given alone as when it was given in combination with 320 milligrams of arsenoxide (42 per cent versus 21 per cent). The combined dose gave the same results as did penicillin alone at à total dosage of $1,200,000$ units.

(b) Penicillin and bismuth.-The failure rate at 160 days with 1,200,000 units of penicillin alone was 10 per cent, which was reduced to 6 per cent when 1 gramme of bismuth was added.

(3) Intervals between injections and the duration of treatment.- There was no evidence of a sizable difference, according to whether the interval between injections was 3 hours or 6 hours, or whether the duration of treatment was 4 days or $7 \frac{1}{2}$ days. There was a slight difference in favour of 3 hours rather than 6 in a course of treatment of $7 \frac{1}{2}$ days.

(4) Duration of disease. - For long duration of the disease the failure rate was higher than for short duration. For duration of 2 months or more, the failure rate at the end of 11 months was more than twice that of cases treated in the first week of the disease (32 per cent against 14 per cent).

(5) Colour and sex. - There was no difference in results between white and coloured races at the end of 11 months. The female failure rate was slightly higher than the male.

(6) Re-treatment. - Patients re-treated because of clincal failure showed a higher cumulative percentage failure rate by the end of 7 months than did those with previously untreated early secondary syphilis receiving the same dosage of penicillin.

An interesting chart was presented showing the results in the United States Public Health Service Clinics, and comparing those given by the use of $2,400,000$ units of penicillin alone with those from one-day fever and arsenic, with 5 days' and 8 days' intravenous drip arsenic, and with the Eagle 12-weeks' system of arsenic and bismuth. The failure rates at 11 months were as follows.

One-day fever plus arsenic

$2,400,000$ units of penicillin

5 and 8 days' arsenic ...

Eagle's 12-weeks' schedule

$$
\begin{aligned}
& \begin{array}{llllllll}
\ldots & \ldots & \ldots & \ldots & \ldots & \ldots & 19 & \text { per cent }
\end{array} \\
& \begin{array}{lllllll}
\cdots & \cdots & \cdots & \ldots & \ldots & \ldots & 15
\end{array}
\end{aligned}
$$

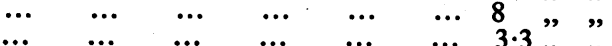

$$
\begin{aligned}
& \begin{array}{lllllll}
\cdots & \cdots & \ldots & \ldots & \ldots & \ldots & 3 \cdot 3,
\end{array}
\end{aligned}
$$


The foregoing represents the collective results of many clinics. Other individual experiences were expressed as follows.

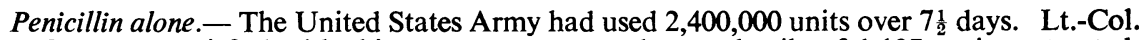
Sternberg was satisfied with this as a treatment and gave details of 1,107 patients treated. About one-third of the number had been followed up for over a year. Seronegative primary cases produced 5.28 per cent of failures, seropositive primary cases 9.54 per cent and secondary cases 17.4 per cent of failures in that time. In only 9 per cent was the treatment considered to be actually unsatisfactory.

Penicillin and arsenoxide.- Dr. Evan Thomas of New York had treated 1,119 patients with $1,200,000$ units of penicillin given 3-hourly over $7 \frac{1}{2}$ days plus 0.04 gramme of Mapharsen daily for 8 days. Of 611 patients followed up, there was a failure rate of 12 per cent at 6 months. With $1,200,000$ units only there was a failure rate of 17.6 per cent in 556 cases followed up for the same time.

Penicillin plus bismuth.- Dr. Schoch (Dallas) had treated 291 cases with 1,200,000 units cf penicillin over $7 \frac{1}{2}$ days plus 1 gramme of bismuth subsalicylate in oil. The failure rate at 160 days was 6 per cent, as compared with 10 per cent in cases in which the same dose of pencillin alone was given.

Penicillin plus fever.- Dr. G. X. Schwemlein (Chicago) described his results with 108 patients treated with $1,200,000$ units of penicillin over $7 \frac{1}{2}$ days plus a three-hours' fever session of $106^{\circ} \mathrm{F}$ (rectal), administered by the use of the hypertherm, on the second, fourth and sixth days. The patients were observed for 154-353 days and there has been a 14.7 per cent failure rate to date. A further 202 patients were given a two-days' treatment, 600,000 units of penicillin being given in two-hourly doses of 50,000 units each on the first day, followed by a six-hours' fever session, during which a further $6 \mathrm{C} 0,000$ units were given in hourly doses of 100,000 units. The patients had been observed for 153-361 days and 19.3 per cent of failures had occurred up to the time of reporting.

Penicillin in oil and beeswax. - Capt. Romansky had treated 68 cases of early syphilis with 8 single daily injections of 300,000 units. Only 22 had been followed up for over 6 months and there had been 2 failures. Major Leifer had treated 200 army cases on the same regimen. He had had 5 instances of secondary fever, 4 of urticaria, 2 of herpes simplex and 2 of disseminated macular eruption on the ninth day. Only 10 of this series had been followed up for over 6 months, and therefore no definite conclusions were drawn.

Technique of treatment with penicillin in oil and wax.-Penicillin in arachis oil and beeswax, which is featured considerably in the new treatment schedules in an attempt to evolve a satisfactory treatment for ambulatory cases, besides being made locally in some centres, is also being made by two commercial houses to the Romansky formula. One of them supplies enough for a single dose in an expendable plastic syringe.

Romansky used calcium penicillin of a potency of 1,000 units per milligram (Romansky and Rittman). He found that sodium penicillin gave unreliable blood levels and also that sodium preparations deteriorated more rapidly than did the calcium salt. The penicillin must be powdered dry, and anhydrous peanut (arachis) oil and sunbleached beeswax must be used. The most effective combination that he had found contained 300,000 units of calcium penicillin in 4.8 per cent beeswax. (by weight) in arachis oil to each 1 cubic centimetre. Patients who received a single injection of 1 cubic centimetre of this preparation all had assayable levels of penicillin in the blood at 12-16 hours, 84 per cent at 20 hours and 70 per cent at 24 hours after the injection. The preparation maintained its potency for over a year at room temperature and refrigerator temperatures. There was no loss at $56^{\circ} \mathrm{C}$ until $24-36$ hours and even at $100^{\circ} \mathrm{C}$ there was no loss for 2 hours. In giving the injection absolute dryness was essential, as moisture caused this preparation to stick in the needle.

At the Walter Reed General Hospital, Washington (D.C.), a vacuum autoclave was used for syringe sterilization, but when this was not available the washing through of the sterilized syringe with alcohol and ether was considered to be sufficient. The preparation should be warmed under the tap or on the sterilizer before use, and 2 different needles should be employed, one for extracting the solution and another for giving the injection. Luer Lock syringes were used. At the Walter Reed Hospital, some 25,000 of these injections had been given in 5 months by nurses. Romansky said that he had no doubt as to the preparation being easy to administer. Other doctors, however, from widely separated areas, had a different opinion on this point. The differences probably arose because the Romansky formula was not being used by them, or, if it was, a dry sterilization technique was not being employed. In Romansky's experience, penicillin in arachis oil, given once each day, produced as satisfactory results with syphilis as did aqueous penicillin in round-the-clock administration. Some evanescent local reactions were noted, but allergic manifestations were not more frequent, in his view, than with the aqueous solution. He had noted that patients suffering from a concomitant epidermophytosis were apt to get a general itching and vesicles of the hands. A new anti-histamine preparation, Benadryl, was being studied here and elsewhere in regard to its apparently favourable effect upon allergic manifestations of penicillin therapy. 
Penicillin given orally.-Penicillin orally was not referred to at the Washington meeting. Dr. Thomas (Belle Vue Hospital, New York) has treated in this way 7 patients, 2 of whom I saw. Dark-field tests were all negative within 24 hours and some by the end of 8 hours. The patients received 25,000 units 3-hourly up to 75 doses.

\section{Future research in the treatment of early syphilis}

The new schedules formulated for future study by the Advisory Committee reflect the present trend of ideas. The smaller dosage levels and time durations up to a week were considered as having been explored. Two exceptions were made : a combination of 2,400,000 units of penicillin with 320 milligrams of Mapharsen plus 0.6 grammes of bismuth, given over 8 days, was recommended for one schedule, whereas personnel of the Army and the Navy were to be given $2,400,000$ units of penicillin plus 1 gramme of bismuth, spread over 9 days. Both of these are intended to contrast with previous schedules without the addition of bismuth. The other 3 schedules for the administration of penicillin in aqueous solution were of 2,400,000 units of penicillin, 9,600,000 units, and 2,400,000 units plus 480 milligrams of Mapharsen and 1 gramme of bismuth, respectively, spread over 15 days. Thus higher dosages, longer duration of treatment and combination with other medicaments are all being tried in order to improve the figures which have been presented. In addition, 3 schedules of penicillin in oil and wax were also recommended : $4,800,000$ units in single daily injections, for 8 days; $9,600,000$ units in single daily injections for 16 days and $9,600,000$ units given in 16 injections twice weekly. All injections are to be of 600,000 units each.

Six other schedules were announced for United States Public Health Centres outside the above scheme, and these show similar trends. Of the 3 schedules for administering aqueous penicillin, all are for dosages of 2,800,000 units of penicillin spread over 14 days: one for penicillin alone, one with 5 injections of Mapharsen and one with 3 injections of bismuth in addition. Of the 3 schedules for penicillin in oil and wax, all have a single daily injection of 300,000 units for 14 days $(4,200,000$ units in all), whereas one has in addition 5 injections of Mapharsen and the other 3 injections of bismuth. On the face of it, it would appear that the latter group is nearer success in its attempts to produce a clear-cut result. It should be remembered, however, that the schedules of the first group are a continuation of those which have comprised the work so far presented, and are therefore to be judged only as part of a whole.

Experimental rabbit syphilis.-Several speakers dealt with experiments with penicillin in rabbit syphilis.

Dr. R. C. Arnold (New York) showed the results of increasing dosages of penicillin given over decreasing periods of time in rabbit syphilis. He concluded that rabbit syphilis could be cured in 12 hours or less, and that additional studies should be undertaken with the object of intensifying and shortening the treatment of the syphilitic patient.

Dr. H. Eagle, however, took the opposite view, He considered that, when dealing with human material, the intensification of the dose was a waste of pencillin, as the surplus would only be thrown away. He stated that arsenic combined with the spirochaete and was, indeed, concentrated many times within it ; therefore its effects were felt for some considerable time after the injection was given. Penicillin, on the other hand, was effective only whilst it was present in the surrounding fluid ; therefore, producing a supereffective concentration of penicillin over a short time (which would be done by increasing the dose) would not be as useful as increasing the duration of an already effective level, by repeating the penicillin dose after an interval.

Dr. Eagle showed (as he had already demonstrated in published work (Eagle, Magnuson and Fleischman)) that penicillin and Mapharsen were synergistic in rabbits and that the amount of penicillin required to cure rabbit syphilis was reduced to a fraction when subcurative doses of Mapharsen were added ; consequently, if the penicillin and bismuth were to prove to be similarly synergistic, the optimum therapy for human syphilis might well consist of penicillin, bismuth and Mapharsen.

Dr. C. M. Carpenter, working on penicillin and fever in rabbit syphilis, found much better results when he used fever combined with multiple rather than with single injections of penicillin; he concluded that the results of penicillin and fever were additive rather than synergistic.

Other research.-In another field Dr. Mahoney (New York), speaking about persistent positive serological reactions without clinical or case-history evidence of syphilis, said that, 
with the exception of pregnant women, patients should not be treated on the basis of serology alone. He believed that soon the new cardiolipid antigen would be available, and that this would change the whole field of serology. With his own patients Dr. Mahoney employed a battery of serological tests in syphilitic cases. A new nomenclature for an improved Lange test was also under preliminary trial in New York. The highest reading in this for a given tube was 18 instead of 5 , as hitherto, and results might be compared by adding the totals.

\section{Treatment of contacts}

An interesting discussion was started at the Bethesda meeting on the question of whether clinically negative contacts of cases of dark-ground-positive cases of syphilis should or should not be treated in the absence of signs of infection. The suggestion to give this treatment received a surprising amount of support, some 50 per cent of those present being in favour: Dr. Evan Thomas had already treated over 50 such cases. He regarded such treatment as necessary for the prevention of "ping-pong" syphilis.

\section{Tertiary syphilis}

Neurosyphilis.-In the field of late syphilis the spirit of research and experimentation is also very evident. The schedules of treatment, however, have not been standardized, and opinions expresssed about the efficacy of penicillin varied. Fever therapy was still employed in many places : at Chicago, for example, neurosyphilis was treated with 12 fever-cabinet sessions, 3 a week for 4 weeks, with 60 milligrams of Mapharsen administered at the height of each fever and 200 milligrams of bismuth given for 4 injections, 1 each week, over the same period. In Philadelphia penicillin was used without pyretotherapy in doses of $4,800,000$ units or less, whereas at the Belle Vue Hospital (New York) 6,000,000 units were given in 150 three-hourly injections; if re-treatment was considered necessary, this dosage was increased to $8,000,000-9,000,000$ units in two-hourly doses. At the Johns Hopkins Hospital, Baltimore, 10,000,000-20,000,000 units were being used in 200 three-hourly injections spread over 25 days. It was the general policy to start immediately with the maximum dose, and although no unusual reactions were noted as the result of this, one reporting centre did state that it was common for paranoid reactions to develop in paresis cases, the exciting factor usually being the hospital "loud speaker".

At the Washington meeting Dr. J. H. Stokes gave details of 197 of a large group of cases which he had kept under observation for more than 120 days.

Treatment was confined to penicillin in dosage levels of $1,200,000-4,800,000$ units and $4,800,000-10,000,000$ units, given by round-the-clock administration in three-hourly intramuscular injections. He found little difference between the two dosage ranges, and said that the most striking signs of improvement were to be found in the cerebrospinal fluid findings, in which they had been consistent, prolonged and progressive. The most obvious changes were found in the first 120 days, but they might continue well after that time. Out of 51 tabetics, 44 were regarded as distinctly improved. From the clinical standpoint, in symptomatic neurosyphilis there was some improvement in 65 per cent (marked in 24 per cent), no change in 26 per cent ; 9 per cent were worse. Four cases with Charcot's joints showed no change and 4 cases showing gastric crisis remained unaffected, although lightning pains responded. There was no apparent concomitance between the changes in the serology of the blood and of the cerebrospinal fluid findings. Of 115 cases with initially seropositive blood tests and showing marked spinal fluid improvement, only 36 (31 per cent) showed marked improvement in the blood. It would almost seem, Dr. Stokes added, as if a different mechanism were involved in the blood response as compared with the spinal fluid.

Sixty cases of optic atrophy had been treated and 19 patients had been seen for a year ; there was spinal fluid improvement in 75 per cent. Of 9 patients who were presumably progressing prior to treatment, 4 showed further visual field contraction, one became blind and 4 showed no change; the spinal fluid improved in all the 8 who were affected. The hypothesis was put forward that each neurone lost in optic atrophy was an irreplaceable unit, whereas the brain had a large functional margin of neurones. It was concluded that a single course of not less than 4,800,000 units in $7 \frac{1}{2}$ days would make the best start and was usually an adequate total therapy for the majority of cases of neurosyphilis. Observations should be carried on for at least a year, in the absence of convincing evidence of progression, before the effects were evaluated and further measures adopted.

At Boston Dr. Rose (who presented results of cases treated at Dr. Solomon's clinic) had taken 176 patients for study, 136 of whom had general paresis. Of these patients 41 received penicillin alone, 37 penicillin plus half a course of fever, and 98 penicillin plus half a course 


\section{THE BRITISH JOURNAL OF VENEREAL DISEASES}

of malaria. (Half a course of malaria was 4-6 paroxyms of fever, and half a course of fever consisted of 20 hours of fever above $105^{\circ} \mathrm{F}$. in the fever cabinet.) Penicillin was given in 6 schedules of 3,000,000-18,000,000 in 5-45 days. Clinical improvement was very noticeable with penicillin and malaria (61 per cent) and with penicillin and fever (56 per cent), whereas with penicillin only it was seen in 41 per cent of the cases.

Dr. W. E. 'Graham, of Hot Springs, reviewed 83 cases observed over 3 months and treated with $6,000,000$ units of oil-beeswax penicillin, patients being given 400,000 units daily for 15 days in conjunction with 8 alternate daily injections of 0.04-0.06 gramme of Mapharsen plus $\mathbf{0 . 2}$ gramme of bismuth every third day for 5 injections. His conclusion was that the clinical and laboratory findings of this treatment at the end of 3 months were comparable with those obtained after fever therapy at the end of 6 months, and that such combined therapy might well replace malaria and fever therapy entirely in the treatment of neurosyphilis.

In contrast with these encouraging reports, Dr. R. R. Kierland, of the Mayo Clinic, described 42 cases treated with up to $16,000,000$ units, some with fever therapy in addition. He concluded that in neurosyphilis penicillin did not achieve the results which it gave in the other forms of the disease; penicillin alone was inferior to penicillin and fever, and when it was given with malaria the results were better than with fever alone. Penicillin was of no value in the "burned-out" tabetic, and patients who had had much treatment with arsenic and bismuth obtained little further benefit from penicillin.

\section{Other forms of tertiary syphilis}

Little was said concerning other forms of late syphilis, although this was a big problem in the South. In a recent blood-testing survey in Savannah (Georgia), when over 71,000 blood tests were taken in a population of 125,000 , some 18 per cent had a positive blood test, due in a large proportion of cases to latent syphilis. Dr. W. J. Brown, when approached as to the disposal of these cases, had decided, in spite of possible dangers from cardiovascular syphilis, that all suitable patients should be admitted for intensive treatment up to the age of 45 years. No serious reactions were encountered.

Cardiovascular syphilis has not come under the light of as intensive an examination as have the other forms which have been mentioned. It had formerly been the practice to treat these cardiovascular lesions with pencillin, starting with the maximum dose. A few mishaps have produced caution, and now the initial dose in cases of angina sometimes is as low as 500 units.

\section{Discussion}

Before concluding these remarks on the present trends of venereal disease treatment in the United States of America, I should like to single out a few points for special discussion. First, in gonorrhoea, the dosage of penicillin employed is higher, on the whole, than that which is generally used here; secondly, the tests of cure are less stringent and the bugbear of masked syphilis is not taken too seriously, in relation either to the initial dose of penicillin employed or to the length of the follow-up period. It would be interesting to collect the views of British venereologists on these points, especially as to how many cases have been seen by them in which syphilis has developed in a patient who has received penicillin treatment for gonorrhoea and in which it has taken longer than 3 months to declare itself. Much that has been said concerning the penicillin treatment of syphilis represents British views also. Perhaps the most controversial subject is that of the treatment of the apparently non-infected paramours.

\section{ACKNOWLEDGEMENT}

I should like to express my very sincere appreciation for the cooperation and hospitality offered by all the American venereologists with whom I came into contact. Their ready response in showing me their clinics and discussing their work was universal, and this opportunity is taken to acknowledge their many kindnesses.

ADDENDUM 1.

Since the above address was given, many articles written by the authorities quoted have appeared in successive numbers of the Journal of the American Medical Association, culminating in the issue of 25th May 1946. This latter, however, contained some new and startling information, which, it is felt, must of necessity appear here (Committee on Medical Research).

In this issue appeared a most important joint statement, entitled "The Changing Character of Commercial Penicillin", by the Committee on Medical Research, United States Health Service and Food and Drug Administration. 


\section{TREATMENT OF VENEREAL DISEASE IN THE UNITED STATES}

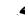

It stated that within a week after the February 6-7th Conference disturbing information becam available from two laboratories.

These laboratories were studying the effect of penicillin $\mathrm{K}$ on rabbit syphilis and found a tenfold difference in efficiency between it and penicillin $G$, the penicillin $K$ being less effective. A few days later another hospital reported that a regimen with 1,200,000 units of penicillin over 33 days had been found paradoxically to be more efficient than one with double the dose over double the time.

This prompted an immediate further analysis of the cases at the Johns Hopkins Hospital, which demonstrated that cases treated before May 1944 became seronegative at 11 months in 85 per cent, whereas of those cases treated after that date, only 60 per cent were seronegative in the same time.

The article then states: "The conclusion seems inescapable that certain commercial penicillins produced within recent months are less efficacious in the treatment of syphilis than were the preparations available two years ago. It is probable that some of the decreased therapeutic effect is due to the increased amount of penicillin $K$ which has been present in the commercial preparations of many manufacturers. The existence of other factors, such as the decrease in the amount of possibly therapeutically active impurities, must also be considered.'

Recommendations follow for the treatment of early syphilis, suggesting 3,600,000 units of penicillin for seronegative primary syphilis and 5,400,000 units for seropositive primary and early secondary syphilis. For relapses a second course of penicillin, plus 360 milligrams of Mapharsen, given in 6 injections, and 6 injections of bismuth, is recommended.

Obviously, this disconcerting information has to be integrated with the data presented at the Washington meeting. Whether or not the schedules proposed for future study have been changed is not at present known.

\section{ADDENDUM 2.}

At the time of going to press I learn in a personal communication from Dr. J. E. Moore of certain very rapid and important steps forward in the penicillin situation.

All the manufacturers in the United States of America have taken immediate steps to reduce the $K$ content of commercial penicillin, with entire success, and all penicillins available now in the United States consist predominantly of penicillin $G$. One product is indeed a crystalline substance containing 95 per cent $G$. It is predicted that within 6 months all penicillins will be pure or almost pure penicillin $\mathbf{G}$.

As from 1st July 1946, the syphilis study group under the auspices of the United States Public Health Services has gone over from commercial penicillin to crystalline penicillin $G$, using four treatment schedules of $7 \frac{1}{2}$ days, giving $2,400,000$ or $4,800,000$ units at two-hourly or threehourly intervals. With commercial penicillin, doses up to 8,000,000 units are now being tried.

\section{REFERENCES}

Committee on Medical Research, The United States Health Service and The Food and Drug Administration. (1946) J. Amer. med. Ass. 131, 271.

Eagle, H., Magnuson, H. J., and Fleishman, R. (1946) J. vener. Dis. Inform., $27,3$. Romansky, M. J., and Rittman, G. E. (1945) New Engl. J. Med., 233, 577.

$A$ portion of the above article is appearing in "Nature".

\section{DISCUSSION ON THE PRECEDING PAPER}

Air-Cdre. G. L. M. McElligott (the President) opened the discussion. With regard to the desirability or otherwise of treating clinically suspicious female contacts in the absence of pathological confirmation, he thought that most people would probably agree that, after an unprotected intercourse with an infected man, the odds on the woman becoming infected with gonorrhoea were very heavy. Some would even say that in such a case it was unnecessary to' wait for the appearance of sign's and symptoms before treatment should be commenced.

With regard to the masking of syphilis by the penicillin treatment of gonorrhoea, he had seen remarkably few cases in which this could be shown to have occurred. Strangely enough, the most convincing case was that of a man whose primary sore appeared. 6 months after he had received 100,000 units of penicillin for gonorrhoea. He was a reliable witness, and his statement, that in the meantime he had not exposed himself to further infection, could be accepted.

Lt.-Col. Willcox said that the Army had been circularized with a view to discovering whether or not a case could be found in which syphilis had declared itself later than 3 months after the finally attested cure of gonorrhoea; so far he had not found anybody who claimed to have seen such a case.

Dr. J. Marshall said that he had seen half a dozen cases of treated gonorrhoea in which syphilis had declared itself later, apparently (according to the patient's statement), without reinfection; in those cases the average incubation period of syphilis was about 50 days. It looked as though the penicillin treatment had delayed the appearance of the signs of the disease, which, when they appeared, were what one would expect; the chancre was normal in appearance 